\title{
ИСПОЛЬЗОВАНИЕ МОДЕЛИ АТОМ-АТОМНЫХ ПОТЕНЦИАЛОВ ПРИ ИССЛЕДОВАНИИ ОСОБЕННОСТЕЙ ВЗАИМОДЕЙСТВИЯ МОЛЕКУЛ В КРИСТАЛЛАХ
}

https://doi.org/10.31241/MIEN.2018.14.10

Геворгян А.А. ${ }^{1}$, Малеев А.В. ${ }^{1}$, Потехин К.А. ${ }^{1}$

${ }^{1}$ Владимирский государственный университет, Владимир, armkir777@gmail.com

\section{Аннотация}

Предложен новый подход к исследованию и описанию молекулярных упаковок в кристаллах, основанный на анализе межмолекулярных парных атом-атомных потенциалов. С использованием этого подхода выявлены различные типы эффективных контактов молекула-молекула, встречающиеся в молекулярных кристаллах органических соединений.

\section{Summary}

A new approach to the study and description of molecular packings in crystals based on an analysis of intermolecular pair atomic-atom potentials is proposed. Using this approach, various types of effective molecular-molecule contacts, found in molecular crystals of organic compounds, have been identified.

\section{Введение}

В настоящее время с помощью рентгендифракционного метода исследования монокристаллов изучено огромное количество кристаллических структур. Результаты этих исследований (около миллиона кристаллических структур) представлены в Кембриджском банке структурных данных [1]. Однако, проблема объяснения взаимного расположения молекул в кристалле друг относительно друга (особенности молекулярной упаковки) все еще остается не полностью решенной. Например, активно дискутируются следующие три вопроса. Во-первых, почему молекулы в конкретной кристаллической структуре располагаются друг относительно друга именно так, а не иначе? Во-вторых, возможны ли полиморфные модификации конкретной кристаллической структуры (иное взаимное расположение молекул друг относительно друга)? В-третьих, для каких кристаллических структур вероятность существования полиморфных модификаций достаточно высока?

Основы развиваемых в настоящее время подходов к поиску ответов на такие вопросы были заложены А.И. Китайгородским [2], который обобщил принцип плотной упаковки на молекулярные кристаллы. Одна из формулировок этого принципа звучит так: “взаимное расположение молекул в кристалле всегда таково, что «выступ» одной молекулы приходится на «впадину» соседней» [2]. Следствием этого принципа с геометрической точки зрения можно считать стремление молекулярной упаковки в молекулярных кристаллах к максимально возможному коэффициенту упаковки (минимуму пустот), а с энергетической точки зрения стремление кристаллической структуры к минимуму энергии межмолекулярного взаимодействия. 
П.М. Зоркий развил идеи А.И. Китайгородского и предложил рассматривать кристаллическую структуру как упаковку молекулярных агломератов, которые представляют собой наиболее прочно связанные ансамбли молекул, включающие в себя конечное или бесконечное число молекул [3]. К конечным агломератам следует отнести пространственно обособленные молекулярные димеры, тримеры, тетрамеры и т. п. Бесконечные агломераты - это молекулярные цепи, молекулярные слои, пространственные образования, бесконечные в трех измерениях. Существование в кристалле молекулярного агломерата предполагает наличие в нем сильного взаимодействия соседствующих молекул, которое вносит значительный вклад в общую энергию кристаллической структуры, называемое эффективным контактом молекула-молекула [3]. Часто наличие эффективных межмолекулярных контактов объясняются большим числом участвующих в его образовании опорных атом-атомных контактов [4], определяемых с использованием системы ван-дер-ваальсовых радиусов атомов [5].

Однако сильное межмолекулярное взаимодействие в агломерате не всегда свидетельствует о его устойчивости вне кристаллической структуры. В большей степени эту устойчивость характеризует жесткость контакта [6], определяемая не столько величиной энергии взаимодействия молекула-молекула, сколько наличием достаточно высоких потенциальных барьеров, препятствующих существенному изменению взаимного расположения и ориентации молекул, образующих контакт, в отсутствии взаимодействия молекул этой пары с другими молекулами кристаллической структуры. Очевидное су-

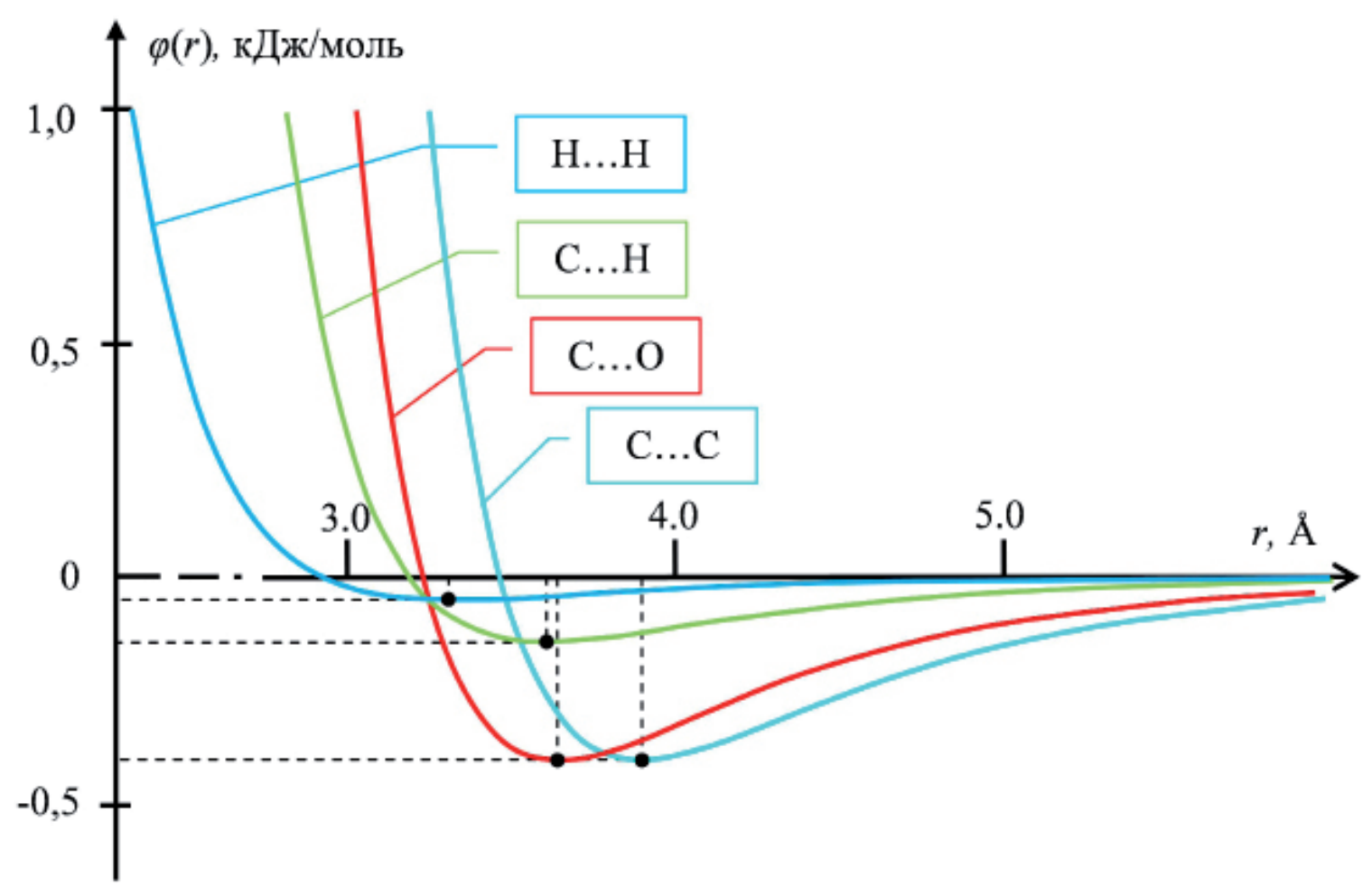

Рис. 1. Потенциальные кривые атом-атомных потенциалов взаимодействия $C \ldots C, C \ldots H, C \ldots O, H \ldots H$. 
ществование глобального или локального минимума энергии межмолекулярного взаимодействия кристаллической структуры в целом не всегда свидетельствует о существовании аналогичных минимумов энергии парного взаимодействия молекула-молекула даже для эффективных контактов. В работе [7] предложен алгоритм оценки жесткости контактов молекула-молекула.

Для оценки энергии межмолекулярного взаимодействий в кристаллах, как правило, используются квантово-химические расчеты [8-10]. Однако, как сами квантово-химические расчеты, так и интерпретация их результатов являются весьма сложной задачей и обычно становятся предметом для активных дискуссий.

Кроме квантово-химических методов оценки энергии межмолекулярного взаимодействия в кристаллах можно использовать метод атом-атомных потенциалов. В работе [11] показано, что использование метода атом-атомных потенциалов является правомерным, т.к. он «оказывается вполне обоснованным из первых принципов квантовой механики».

В этой работе мы рассматриваем некоторые приемы использования модели атом-атомных потенциалов для анализа межмолекулярного взаимодействия в кристаллических структурах органических соединений.

\section{Методы исследования}

В рамках модели атом-атомных потенциалов энергия межмолекулярного взаимодействия двух молекул в димере определяется как сумма $U_{M-M}=\sum_{i, j} \varphi_{i j}$ атом-атомных потенциалов $\varphi_{i j}=-A_{i j} r_{i j}^{-6}+B_{i j} \exp \left(-C_{i j} r_{i j}\right)$. Параметры $A_{i j}, B_{i j}, C_{i j}$ взаимодействия $i$-го атома первой молекулы с $j$-ым атомом второй молекулы определяются типами этих атомов по формулам $A_{i j}=\sqrt{A_{T_{i}} A_{T_{j}}}, B_{i j}=\sqrt{B_{T_{i}} B_{T_{j}}}$ и $C_{i j}=\sqrt{C_{T_{i}} C_{T_{j}}}$ (параметры $A_{T}, B_{T}$ и $C_{T}$ для типов атомов $\mathrm{C}, \mathrm{H}, \mathrm{O}$ и $\mathrm{N}$ взяты нами из работы [12]). Таким образом, энергия взаимодействия двух конкретных атомов зависит только от расстояния $r_{i j}$ между ними, т.е. является функцией $\varphi(r)$ одной переменной (потенциальная кривая).

На рис. 1. представлены четыре потенциальные кривые для четырех наиболее распространенных в органических соединениях типов межмолекулярного атом-атомного взаимодействия: С...С, С ...Н, Н...Н и С...О. Потенциальные кривые имеет минимум $\frac{\partial \varphi\left(r_{0}\right)}{\partial r}=0$, которому соответствует равновесное расстояние $r_{0}$ между атомами. Минимум потенциала - глубина потенциальной ямы - практически совпадают для контактов С...С и С...О $\varphi_{0}=0.34$ кДж/моль. Для контактов типа С...Н составляет примерно $36 \%$ от этого значения, а для контактов типа Н...Н - менее 14 \%, поэтому в первом приближении при описании межмолекулярного взаимодействия можно ограничиться только анализом атом-атомных потенциалов между неводородными атомами. Каждому типу взаимодействия соответствует свое равновесно расстояние $r_{0}$ (табл.1). Как и следовало ожидать, наименьшее расстояние $r_{0}$ наблюдается для контакта Н...Н, а наибольшее - для контакта C...C. 


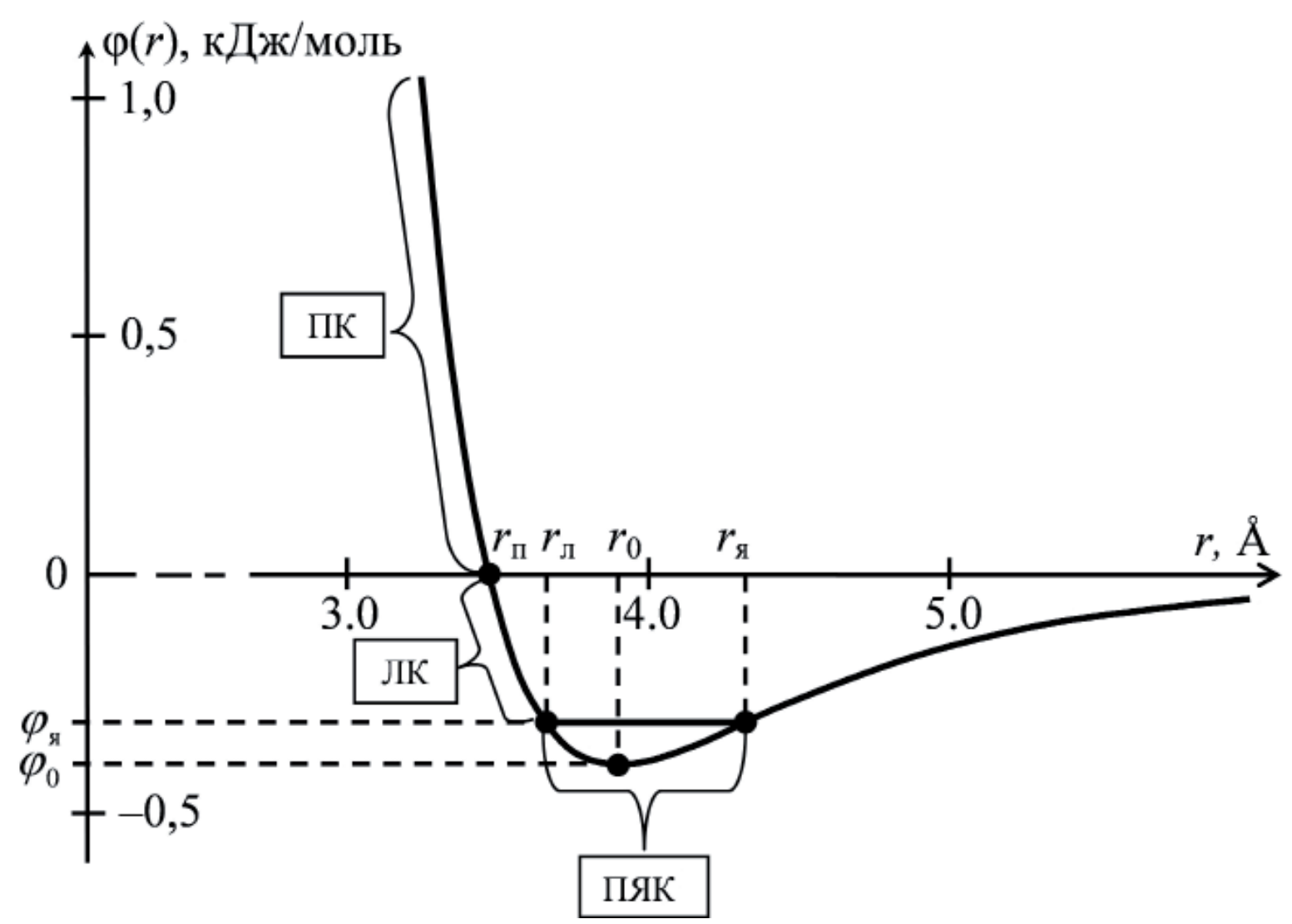

Рис. 2. Особые точки и области кривой атом-атомного потенциала взаимодействия двух атомов углерода.

Для более детальной характеристики межмолекулярных атом-атомных контактов введем еще несколько понятий и величин. Каждая потенциальная кривая имеет точку перегиба $\frac{\partial^{2} \varphi\left(r_{n}\right)}{\partial r^{2}}=0$. Предлагаем рассматривать точку перегиба потенциальной кривой как правую границу потенциальной ямы. Это точка характеризуется параметрами $\varphi_{я}$ (потенциал на правой гра-

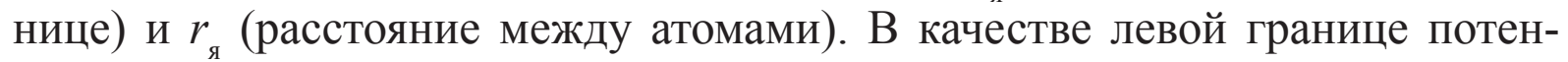
циальной ямы, которой соответствует расстояние между атомами $r_{\text {л }}$, будем рассматривать точку потенциальной кривой, расположенную левее минимума и дающую такое же значение потенциала $\varphi\left(r_{\text {л }}\right)=\varphi_{\text {я. }}$ Еще одна критическая точка определяется обращением в ноль потенциала. Ей соответствует расстояние $r_{\text {я }}: \varphi\left(r_{\text {п }}\right)=0$. Смена знака потенциала физически означает смену энергии притяжения $(\varphi<0)$ на энергию отталкивания $(\varphi>0)$, что соответствует понятию опорного контакта по Зоркому [3]. Численные значения $r_{\text {п }}$ достаточно хорошо согласуются с расстояниями, определяемыми вандер-ваальсовыми радиусами атомов, которое рассчитывается по формуле $r_{\mathrm{B}}=2 \sqrt{R_{i} R_{j}}$, где $R_{i}$ и $R_{j}$ - ван-дер-ваальсовы радиусы $i$-го и $j$-го атомов [5]. Параметры $\varphi_{0}, \varphi_{я}, r_{0}, r_{я}, r_{\text {л }}$ и $r_{\text {в }}$ для четырех потенциальных кривых представлены в табл. 1. На рис. 2. показаны особые точки и области потенциальной кривой на примере взаимодействия двух атомов углерода. 
Таблица 1. Энергетические и геометрические характеристики четырех типов межатомных взаимодействий в модели атом-атомных потенциалов.

\begin{tabular}{|c|c|c|c|c|c|c|c|}
\hline $\begin{array}{c}\text { Тип } \\
\text { контакта }\end{array}$ & $\varphi_{0}, \frac{\mathrm{\kappa Д}}{\text { моль }}$ & $r_{0}, \AA$ & $\varphi_{9}, \frac{\mathrm{KД}}{\text { моль }}$ & $r_{g}, \AA$ & $r_{\mathrm{\pi}}, \AA$ & $r_{\pi}, \AA$ & $r_{\mathrm{B}}, \AA$ \\
\hline C...C & -0.398 & 3.90 & -0.310 & 4.32 & 3.66 & 3.47 & 3.42 \\
\hline C...H & -0.143 & 3.61 & -0.111 & 4.01 & 3.14 & 2.97 & 2.82 \\
\hline $\mathrm{H} \ldots \mathrm{H}$ & -0.054 & 3.31 & -0.041 & 3.70 & 3.08 & 2.91 & 2.32 \\
\hline C...O & -0.399 & 3.64 & -0.311 & 4.04 & 3.41 & 3.24 & 2.96 \\
\hline
\end{tabular}

Рассмотренные критические расстояния разбивают потенциальную кривую на несколько областей. Будем называть атом-атомный контакт контактом, соответствующим потенциальной яме (обозначать аббревиатурой ПЯК - в потенциальной яме контакт), если для него выполняется условие

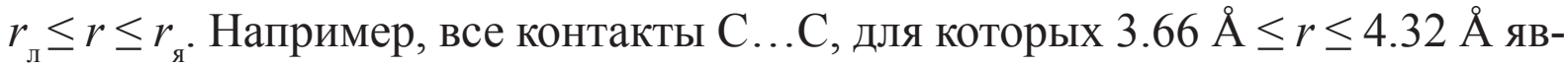
ляются ПЯК. Аналогично для контакта С...Н условием ПЯК является 3.14 $\AA \leq r \leq 4.01 \AA$. Контакты, попадающие в левую часть потенциальной кривой $\left(r_{\text {п }} \leq r \leq r_{\text {л }}\right)$, при условии, что выполняется $0 \leq|\varphi| \leq\left|\varphi_{я}\right|$ будем называть левым контактом и обозначать аббревиатурой ЛК. Контакты с положительным атом-атомным потенциалом $\varphi>0\left(r<r_{\text {п }}\right)$ назовем положительными контактами (ПК).

Межмолекулярные атом-атомные контакты, являющиеся ПЯК, наиболее сильные энергетические контакты, поэтому количество ПЯК, возникающих между двумя соседними молекулами, можно использовать для характеристики энергоемкости и устойчивости контакта молекула - молекула. Если в молекулярном димере обнаруживаются ЛК или ПК, то это может быть, либо вынужденный контакт, который наблюдается в тех случаях, когда суммарная энергия становится существенно больше по абсолютной величине при сокращении одного из контактов, либо межмолекулярное взаимодействие имеет весьма существенное дополнительное специфическое взаимодействие (водородный связь, $\pi$-стекинг, Т-стекинг, галоген-галоген). Так как специфические взаимодействия зачастую невозможно описывать в рамках модели атом-атомных потенциалов, то мы можем говорить, что наличие ЛК или ПК позволяют выявлять такие специфические контакты.

\section{Результаты и их обсуждение}

Для анализа и описания особенностей межмолекулярного взаимодействия мы предлагаем такую характеристику атомов как их активность в парных атом-атомных взаимодействиях. Контакты ПЯК, ЛК и ПК будем называть эффективными атом-атомными контактами, а атомы, участвующие в этих контактах активными атомами. Активность атома можно оценить по суммарному количеству ПЯК, ЛК и ПК, в которых он участвует. Тогда все атомы можно разделить на три группы: неактивные, активные и очень активные. У неактивных атомов ПЯК, ЛК и ПК просто нет. Для активных атомов суммарное количество ПЯК, ЛК и ПК больше нуля и меньше десяти, а 


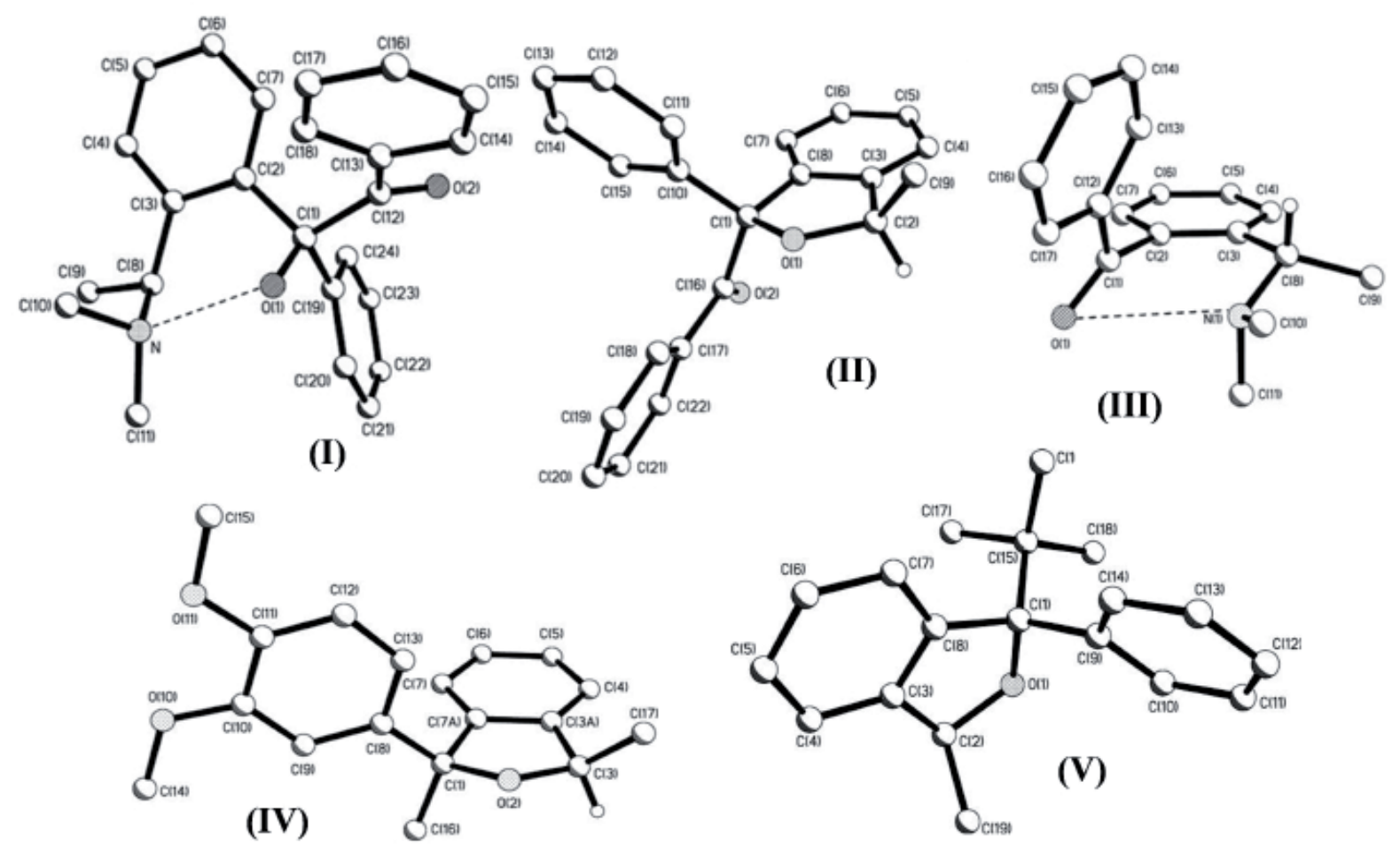

Рис. 3. Перспективный вид молекул I-V с нумераиией неводородных атомов. Внутримолекулярная Н-связь типа O-H...N показана.

атомы, имеющие больше десяти эффетивных атом-атомных контактов, являются очень активными. Очевидно, что деление атомов на активные и очень активные носит условный характер и для разных классов соединений может определяться по-разному.

Для иллюстрации возможностей и особенностей использования предлагаемого подхода проведено исследование кристаллических структур пяти органических соединений. Перспективные виды молекул этих соединений с нумерацией неводородных атомов представлены на рис. 3 .

Все структуры одноорбитные, поэтому для анализа межмолекулярного взаимодействия для каждой из структур были проведены расчеты атоматомных потенциалов для всех молекулярных димеров, образованных базисной молекулой с молекулами своей первой координационной сферы. В таблице 2 представлены результаты анализа активности участия неводородных атомов в межмолекулярном взаимодействии. Во всех пяти структурах подавляющее большинство неводородных атомов являются активными. Неактивных и очень активных атомов либо нет, либо их число не превосходит $20 \%$ от общего числа атомов в молекуле.

Использование модели атом-атомных потенциалов позволяет проводить анализ межмолекулярного взаимодействия с разным уровнем общности. Анализ парных атом-атомных контактов позволяет оценить, как было указано выше, активность отдельных атомов. Но зачастую несколько валентно связанных атомов, входящих в геометрически обособленный фрагмент молекулы (фенил, арил, метоксикарбонил и т.д.) имеет несколько контактов с 
одним атомом соседней молекулы или несколькими атомами, входящими в аналогичный фрагмент соседней молекулы. Тогда можно представить взаимодействие молекул в димере не как сумму атом-атомных контактов, а как совокупность более сильных взаимодействий фрагментов одной молекулы с отдельными атомами или целыми фрагментами другой молекулы этого димера.

Таблица 2. Анализ активности атомов кристаллических структур 1-5.

\begin{tabular}{|c|c|c|c|c|c|}
\hline 空 & $\begin{array}{c}\text { Рефкод } \\
\text { структуры }\end{array}$ & $\begin{array}{c}\text { Количество нево- } \\
\text { дородных атомов } \\
\text { в молекуле }\end{array}$ & $\begin{array}{c}\text { Количество } \\
\text { неактивных } \\
\text { атомов }\end{array}$ & $\begin{array}{c}\text { Количество } \\
\text { активных } \\
\text { атомов }\end{array}$ & $\begin{array}{c}\text { Количество } \\
\text { очень активных } \\
\text { атомов }\end{array}$ \\
\hline I & LOFLIB & 27 & 4 & 21 & - \\
\hline II & COTLEE & 24 & - & 18 & 3 \\
\hline III & NELQOL & 19 & 1 & 18 & - \\
\hline IV & MIFPIB & 24 & 1 & 21 & 2 \\
\hline V & RUFRUG & 20 & 4 & 16 & - \\
\hline
\end{tabular}

Так в кристаллической структуре II обнаружены два межмолекулярных контакта, которые образуют атомы углерода С(17) и С(14) метильных групп с плоскими фрагментами соседних молекул (рис. 4 а и б). Оба эти межмолекулярные контакта содержат по семь атом-атомных контактов, являющихся ПЯК. Это атом-атомные контакты с атомами С(3АA), C(4A), C(5A), C(6A), C(7B), C(7AA), С(16А) в первом случае, и с атомами О(2), C(1), C(8), С(9), $\mathrm{C}(10), \mathrm{C}(13), \mathrm{C}(16)$ во втором. Очень похожий контакт образует атом $\mathrm{C}(6 \mathrm{~A})$ фенильной группы с плоским фрагментом соседней молекулы в кристаллической структуре III.

Рассмотрим еще одну особенность взаимодействия молекул, достаточно часто встречающуюся в органических кристаллах. Если атомы фрагмента молекулы имеют множественные эффективные атом-атомные контакты с атомами двух фрагментов соседней молекулы, которые словно клешнёю

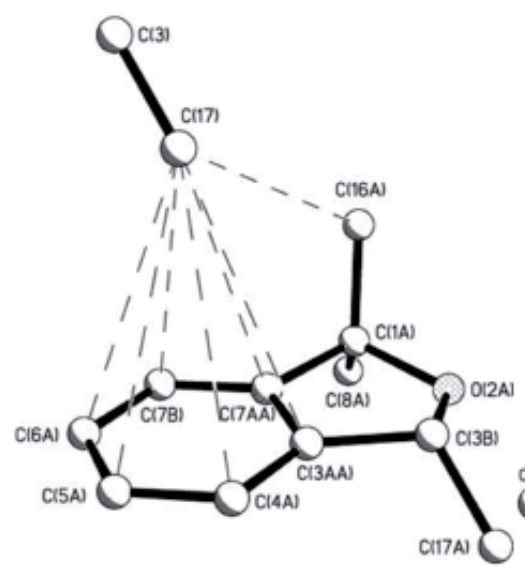

(a)

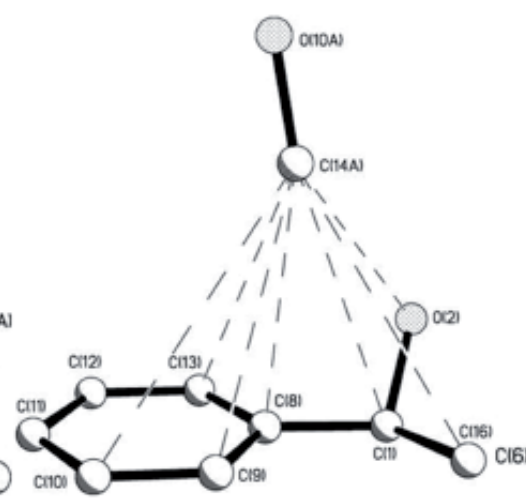

(б)

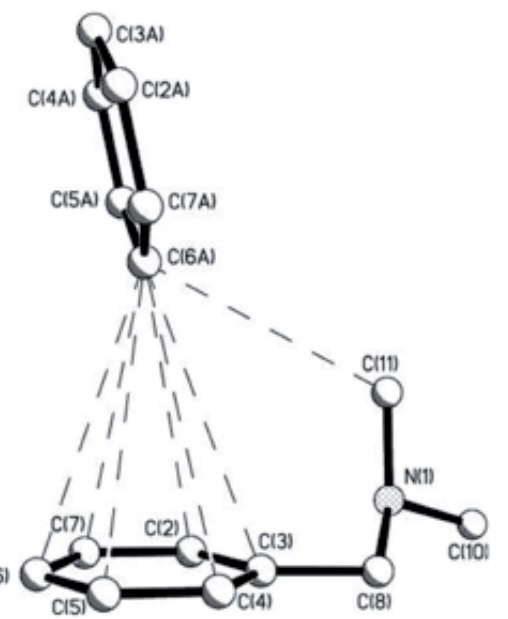

(B)

Рис. 4. Примеры взаимодействия активных атомов с фрагментами соседних молекул в структуре II (а), (б) и в структуре III (в). 


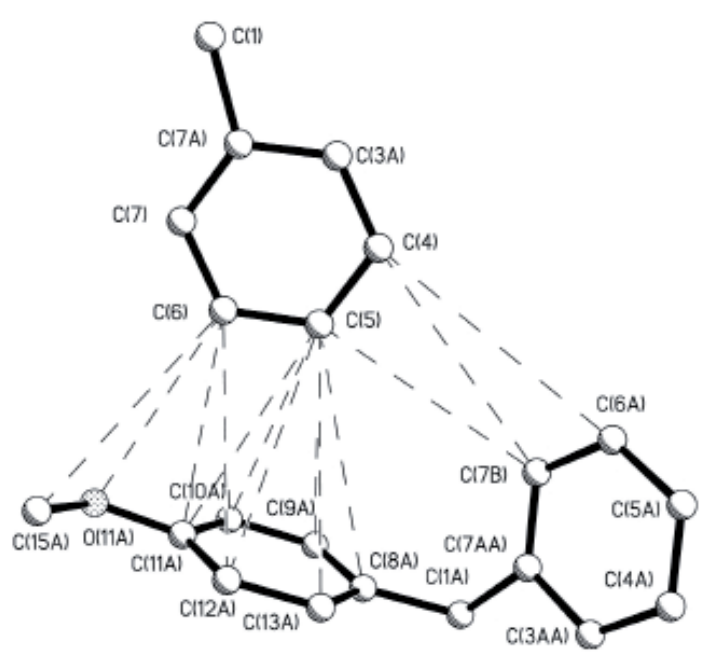

(a)

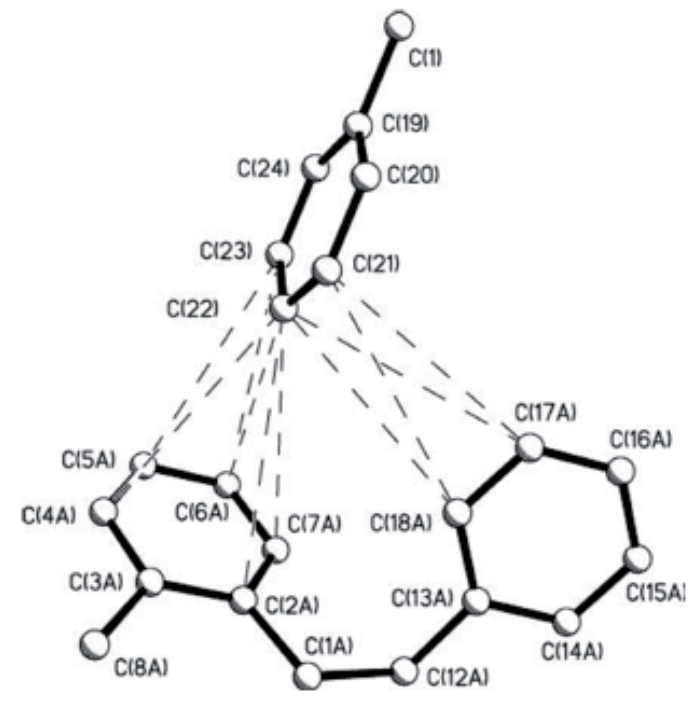

(б)

Рис. 5. Примеры «хелатных ловушек» в структуре II (а) и в структуре I (б)

удерживают этот фрагмент. Будем называть такой межмолекулярный контакт «хелатной ловушкой». Так в кристаллической структуре II атомы углерода С(4), С(5), С(6) фенильного кольца удерживаются эффективными контактами, которые образуют эти атомы с арильным (11 ПЯК) и фенильным (3 ПЯК) фрагментами соседней молекулы (рис. 5 a). Аналогичная «хелатная ловушка» была обнаружена в структуре I. Атомы C(21), C(22), C(23) образуют эффективные атом-атомные контакты с фенильным (4 ПЯК) и арильным (6 ПЯК) фрагментами соседней молекулы (рис. 5 б).

\section{Выводы}

Показана возможность использования особенностей потенциальной кривой (ПЯК, ЛК и ПК) и анализа межмолекулярных атом-атомных контактов между отдельными фрагментами молекул для исследования и описания супрамолекулярной структуры молекулярных кристаллов. Анализ активности атомов в образовании межмолекулярных контактов позволяет представить взаимодействие молекул в димере двух соседних молекул не как сумму атом-атомных контактов, а как совокупность более сильных взаимодействий фрагментов одной молекулы с отдельными атомами или целыми фрагментами другой молекулы этого димера.

Работа выполнена при финансовой поддержке РФФИ, гранты 17-0200835-а и 17-42-330787.

\section{Список литературы}

1. Cambridge Structural Database. Version 5.38. University of Cambridge, UK.

2. Китайгородский А.И. Молекулярные кристаллы. М: Наука, 1971. 424 с.

3. Зоркий П.М., Зоркая О.Н. // Журн. структур. химии. 1998. № 1. Т. 39. С. 126.

4. Зефиров Ю.В., Зоркий П.М. // Журн. структур. химии. 1974. № 1. Т. 15. C. $118-122$. 
5. Зефиров Ю.В., Зоркий П.М. // Успехи химии. 1989. № 5. Т. 58. С. 713-746; 1995. № 5. T. 64. С. 446-460.

6. Зоркий П.М., Зоркая О.Н. // Журн. структур. химии. 2000. № 5. Т. 41. C. 1054-1066.

7. Малеев А.В., Седов Б.Б., Житков И.К., Рау В.Г. // Журн. структур. химии. 2007. № 1. Т. 48. С. 148.

8. Shishkin O.V., Dyakonenko V.V., Maleev A.V., Schollmeyer D., Vysotsky M.O. // CrystEngComm. 2011. T. 13. P. 800.

9. Shishkin O.V., Dyakonenko V.V., Maleev A.V. // CrystEngComm. 2012. T. 14. P. 1795.

10. Shishkin O.V., Zubatyuk R.I., Maleev A.V., Boese R. // Structural Chemistry. 2014. T. 25. № 5. Р. 1547.

11. Грибов Л.А. // Журн. структур. химии. 2005. Т. 46. № 2. С. 311.

12. Williams D.E., Houpt D.J. // Acta Crystallographica. 1986. B 42. P. 286.

\section{ОЦЕНКА ЧИСЛА p2-РАЗБИЕНИЙ ПЛОСКОСТИ НА ПОЛИГЕКСЫ} ЗАДАННОЙ ПЛОЩАДИ

https://doi.org/10.31241/MIEN.2018.14.11

Шутов А.В. ${ }^{1}$, Коломейкина Е.В. ${ }^{2}$

${ }^{1}$ Владимирский государственный университет, Владимир, a1981@mail.ru

${ }^{2}$ МГТУ им. Н.Э. Баумана, Москва, pihta2@rambler.ru

\section{Аннотация}

В работе рассматривается задача о числе $p 2$-разбиений плоскости на полигексы заданной площади. То есть таких разбиений, при которых любую фигуру разбиения можно перевести в любую другую фигуру этого разбиения параллельным переносом либо центральной симметрией, причем преобразование переводит все разбиение в себя. Пусть $t(n)$ - число $p 2$-разбиений плоскости на полигексы площади $n$. Доказано, что справедливо неравенство $C_{1} \cdot 2^{n} \leq t(n) \leq C_{2} \cdot n^{5} \cdot 3,42^{n}$. При доказательстве нижней оценки использована явная конструкция, позволяющая построить требуемое число $p 2$-разбиений плоскости. Доказательство верхней оценки основано на критерии Конвея существования $p 2$-разбиений плоскости и на теории самонепересекающихся блужданий на гексагональной решетке.

Полигекс представляет собой связную фигуру на плоскости, составленную из конечного числа единичных правильных шестиугольников, примыкающих друг к другу по целым сторонам. Полигексы можно рассматривать как конечные подмножества шестиугольного паркета со связной внутренностью. Название полигекс было предложено Д. Кларнером [18]. Полигексы изучались в работах Гарднера [1-4], Голомба [5], Роадса [24], Майерса [22], Малеева $[6,21]$, Факуды $[14,15]$ и других авторов. Большой вклад в 